\title{
Establecimiento de las ventajas de las redes sociales como estrategia de comercialización en las empresas del sector servicios de fumigación
}

\author{
Felipe Alonso Aguilar Topete \\ faguilar2@ucol.mx \\ https://orcid.org/0000-0002-3043-6847 \\ Universidad de Colima, Colima, México \\ Cruz Osbaldo Sánchez Figueroa \\ cruzfigueroa@ucol.mx \\ https://orcid.org/0000-0002-1462-3132 \\ Universidad de Colima, Colima, México
}

\section{RESUMEN}

El presente trabajo tiene como principal objetivo "identificar si los factores como las redes sociales, la periodicidad y las ofertas aumentan las ventas del sector servicios de fumigación", gracias a los resultados obtenidos y el análisis elaborado se pudieron lograr los objetivos y reafirmarlos.

De acuerdo al objetivo que se planteó al inicio tal como identificar aplicando métodos estadísticos descriptivos y análisis factoriales, las principales relaciones que existen entre las redes sociales, la periodicidad y las ofertas con el aumento de ventas de las empresas de fumigación en el estado de Colima.

Con los resultados obtenidos y el estudio realizado se pudieron comprobar las hipotesis, al establecer una correlación significativa con cada una de las variables independientes como redes sociales, periodicidad y ofertas, comprobando que son factores esenciales para lograr aumentar las ventas en las empresas de fumigación.

Por lo tanto las variables independientes tales como redes sociales, periodicidad y ofertas tienen una correlación significativa y se cumple en un 100\% con base al análisis factorial que se hizo para este tipo de estudio, culminando con la fórmula que se obtuvo al hacer este análisis factorial: fórmula $\mathbf{y}=\mathbf{a}+\mathbf{x b}$ por lo que cada que aumente el coeficiente $B$ se verá un aumento proporcional de las demás variables.

Palabras clave: redes sociales; publicidad: marketing: periodicidad: ventas. 


\title{
Establishment of the advantages of social networks as a marketing strategy in companies in the fumigation services sector
}

\begin{abstract}
The main objective of this work is to "identify whether factors such as social networks, periodicity and offers obtained sales in the fumigation services sector", thanks to the results obtained and the analysis prepared, the objectives could be achieved and reaffirmed.

According to the objective that was raised at the beginning, such as identifying by applying descriptive statistical methods and factor analysis, the main relationships that exist between social networks, the periodicity and the offers with the increase in sales of the fumigation companies in the state of Colima.

With the results obtained and the study carried out, the hypotheses could be verified, by establishing a significant correlation with each of the independent variables such as social networks, periodicity and offers, verifying that they are essential factors to achieve increased sales in the fumigation companies.

Therefore, the independent variables such as social networks, periodicity and offers have a significant correlation and it is $100 \%$ fulfilled based on the factor analysis that was done for this type of study, culminating with the formula that was obtained when doing this analysis. factorial: formula $y=a+x b$ so each increase in coefficient $B$ will show $a$ proportional increase in the other variables.
\end{abstract}

Keywords: social networks; advertising; marketing; periodicity; sales.

Artículo recibido: 30 noviembre. 2021 Aceptado para publicación: 29 diciembre 2021

Correspondencia: faguilar2@ucol.mx Conflictos de Interés: Ninguna que declarar 


\section{INTRODUCCIÓN}

El presente trabajo de tesis tiene como principal objetivo identificar aplicando métodos estadísticos descriptivos y análisis factoriales, las principales relaciones que existen entre las redes sociales, la periodicidad y las ofertas con el aumento de ventas de las empresas de fumigación en el estado de Colima.

De acuerdo con el último informe Digital 2021 de Hootsuite y We Are Social, en el cual se recogieron las principales tendencias digitales y el uso que hacen los usuarios de Internet en todo el mundo, los usuarios de Internet a nivel mundial han aumentado en más de 330 millones en el último año, esto reafirma el hecho de que es importante que todas las empresas tengan presencia en redes sociales.

Es importante conocer las necesidades del cliente a nivel local y nacional ya que son ellos los que van a prescindir de tus productos o servicios, muchas veces se comete el error de solamente utilizar la publicidad tradicional.

En el sector de servicios de fumigación es de vital importancia la publicidad por redes sociales, ya que no es un producto tangible, que las personas puedan ver o tocar y decidirse a adquirir, si no que es el prestigio o la buena promoción que se haya generado respecto a la marca, lo que definirá la elección del cliente a elegirte a ti en lugar de a la competencia.

Es por ello la importancia de realizar esta investigación en donde se tomarán en cuenta distintos medios de comunicación (redes sociales) y se describe a detalle el gran impacto que estas alcanzan a nivel mundial.

Para que alguna empresa o negocio obtenga las utilidades deseadas o supere las ya obtenidas, será de vital importancia que se cuente con herramientas de promoción como son las redes sociales.

Según Gallego (2010), define red social como "Conjunto de individuos que se encuentran relacionados entre sí, las relaciones de los usuarios pueden ser de muy diversa índole, y van desde los negocios hasta la amistad".

Para Kirpatrick (2011), las redes sociales, "Son un conjunto de herramientas y mecanismos que proveen una alternativa de hacer marketing de una manera más poderosa", y entre las redes sociales más usadas se encuentran: Facebook, Whats App, Tiktok, Instagram y YouTube. 
Según Rodríguez (2014) "Las Redes Sociales como Facebook, Twitter, LinkedIn, Instagram son una nueva forma de comercializar un producto/servicio".

La publicidad es una herramienta muy importante para dar a conocer cualquier tipo de mensaje. "La publicidad es considerada como una de las más poderosas herramientas de la mercadotecnia, específicamente de la promoción, que es utilizada por empresas, organizaciones no lucrativas, instituciones del estado y personas individuales, para dar a conocer un determinado mensaje relacionado con sus productos, servicios, ideas u otros, a su mercado objetivo" (Thompson, 2015).

Para Giraldo (2019), "La publicidad es la herramienta tradicional directa del marketing, y tiene los objetivos de divulgar un producto para estimular su consumo, transmitir un mensaje positivo con relación a una marca y fortalecer la presencia de una empresa en el mercado".

Lamberton \& Stephen (2016) mencionan que, "La publicidad es adoptada por las empresas como un medio para crear conciencia, ventas y lealtad del cliente". Sin embargo, en los últimos años la forma de hacer publicidad ha cambiado y es que, así como la tecnología avanza, también debe avanzar la forma en la que una empresa hace publicidad.

Cada red social tiene su propia plataforma mediante la cual las empresas y marcas pueden realizar sus campañas publicitarias. Mientras que las grandes empresas gastan millones de dólares en publicidad, las pequeñas empresas a menudo ven la publicidad como un gasto y no como una inversión o estrategia para generar ingresos.

"Hay muchas empresas que hoy en día pasan por esta situación y es porque aún no están dirigidos al sector de mercado acorde al producto que ofrece, la publicidad se encarga de este punto y permite abrir puertas a través de sus mensajes” (Vargas, 2015).

De acuerdo con los autores Soengas, Vivar y Abuín (2015) “El consumidor cada día exige nuevas estrategias de comunicación con las pequeñas y medianas empresas, lo que provoca que estás busquen nuevos formatos para alcanzar mayor audiencia".

Relacionado con lo anteriormente expuesto, debemos hacer mención del "Marketing digital", que lo definiremos como: "El concepto por el cual nos referimos a una serie de herramientas que puestas en acción abren distintos canales de comunicación en el mundo digital." (Genwords, 2019). 
Cuando se utilizan diversas técnicas se pueden idear muchas oportunidades de modelos de negocios y gran cantidad de estrategias, orientadas a detectar oportunidades en los mercados mundiales, para lo cual exigen que las empresas que quieran incursionar en el mercado global desarrollen métodos de comunicación que integren un plan de mercadeo, para posteriormente las empresas puedan segmentar sus mercados y conocer los medios sociales que se utilizan en cada país para promocionar su producto. "Los profesionales en el campo del marketing digital consideran que si un producto o servicio no se encuentra en internet simplemente no existe" (Samaniego, Mejía, y Zambrano, 2018).

Para Ivoskus (2009) citado en Samaniego et al (2018) "El marketing digital permite una integración entre los diferentes medios, aprovechando las posibilidades que nos brindan los nuevos medios junto a los tradicionales, generando así mayor interacción y atrayendo a los usuarios a la participación”. Y es que en combinación de nuevos métodos con los tradicionales se puede llegar a un mercado más amplio y con esto generar mayores ingresos.

Joe Pulizzi (2007) citado en Salas (s.f) definía el marketing de contenido como "Una técnica de marketing de creación y distribución de contenido relevante y valioso para atraer, adquirir y llamar la atención de un público objetivo bien definido, con el objetivo de impulsarlos a ser futuros clientes".

Para Ramos (2016) lo definía como "La creación, publicación y distribución de contenido de un original valor e interés para los clientes, seguidores, fans y comunidad de usuarios". Sin embargo, dicho contenido debe contener calidad e información relevante, que llame la atención del público al que se quiera llegar.

Las tendencias del marketing digital que persisten en la actualidad según Econsultancy State of Ecommerce in China, Amiri \& Woodside (2017), refieren que tras analizar las estadísticas de marketing que están mas a la vanguardia, "Siempre existirán ventajas a la hora de planear y establecer estrategias", como se puede observar en la Grafica \#2, el "marketing de contenidos" tuvo un mayor impacto en el año 2017 con un porcentaje del $22 \%$, en segundo lugar tenemos el "Big Data" con un 17\%, en tercer lugar el "marketing de automatización" con el 13\% y en cuarto lugar el "marketing móvil" con un 12\% respectivamente.

En la actualidad "Los smartphones están teniendo un papel destacado en cuanto a inmediatez" (John \& Kumar, 2015). "Ya no podemos vivir sin ellos", afirma Krum 
(2010), la mayoría de las personas estarían descontroladas y les resultaría difícil imaginar la vida sin ellos.

Para tal efecto se planteo la siguiente interrogante de investigación ¿Si las redes sociales, la periodicidad y las ofertas aumentan las ventas de las empresas de fumigación en el Municipio de Colima y Villa de Álvarez?

\section{ESTRATEGIAS METODOLÓGICAS}

El diseño de esta investigación es de tipo correlacional según Hernández, Fernández \& Baptista (2007) y Cancela et al (2010), ya que el propósito de esta investigación es conocer el grado de asociación entre la variable independiente con la variable dependiente, es decir; Como las redes sociales, las ofertas y la periodicidad (V.I) aumentan las ventas (V.D.) de las empresas de fumigación en el estado de Colima.

El método que se utilizará en esta investigación es el método cuantitativo según Tamayo y Tamayo (2005) y Bernal (2010), ya que se aplicará una encuesta para conocer la relación que existe entre la variable independiente con la variable dependiente.

La muestra que se utilizo fue de 20 empresas 8 del municipio de Colima y 16 de Villa de Álvarez, el tipo de muestreo probabilistico fue aleatorio simple. Para el instrumento se utilizó una encuesta con 18 preguntas de escala de likert cuya confiabilidad para validar la consistencia del mismo se utilzó el Alpha de Cronbach cuyo resultado fue de $\boldsymbol{\alpha}=\mathbf{0 . 8 2 2}$, según George y Mallery (2003, p. 231) considerado como un coeficiente bueno, dicho tratamiento se hizo en el programa SPSS versión 25.

Para el tratamiento del analisis se utilizó el análisis de componentes principales para reducir sus dimensiones (Sánchez, 2012). Y un análisis descriptivo para obtener información precisa, sencilla, clara y ordenada de los datos (Cognodata, 2019).

\section{RESULTADOS Y DISCUSIÓN}

Con relación a los resultados para dar respuesta a la interrogante con relación si los factores como redes sociales, periodicidad y ofertas generan una relación con el aumento de las ventas en las empresas objeto del estudio con su giro de fumigación.

Por lo que se presenta el análisis descriptivo de los 18 ítems (tabla 1), de la cuales se da una breve reseña. 
Tabla 1. Análisis Descriptivo

\begin{tabular}{|c|c|}
\hline Análisis Descriptivo & $\begin{array}{l}\text { Porcentaje } \\
\text { acumulado }\end{array}$ \\
\hline $\begin{array}{l}\text { 1. ¿Considera que publicar sus servicios en redes sociales es } \\
\text { importante? }\end{array}$ & Muy de acuerdo $70 \%$ \\
\hline $\begin{array}{l}\text { 2. ¿Considera que publicar sus servicios en redes sociales atrae } \\
\text { clientes? }\end{array}$ & Muy de acuerdo $70 \%$ \\
\hline $\begin{array}{l}\text { 3. ¿Cree que las redes sociales aumentan sus ventas de servicios } \\
\text { de fumigación? }\end{array}$ & Muy de acuerdo $55 \%$ \\
\hline $\begin{array}{l}\text { 4. ¿Considera que han aumentado sus ventas desde que ofrece } \\
\text { sus servicios en redes sociales? }\end{array}$ & De acuerdo $50 \%$ \\
\hline $\begin{array}{l}\text { 5. ¿Qué tan de acuerdo está en que las empresas de fumigación } \\
\text { utilicen las redes sociales para aumentar sus ventas? }\end{array}$ & Muy de acuerdo $80 \%$ \\
\hline $\begin{array}{l}\text { 6. ¿Considera que es importante publicar periódicamente } \\
\text { contenido acerca de su empresa de fumigación? }\end{array}$ & De acuerdo $75 \%$ \\
\hline $\begin{array}{l}\text { 7. ¿Considera que periodizar el contenido de sus publicaciones } \\
\text { ayude a aumentar sus ventas? }\end{array}$ & De acuerdo $50 \%$ \\
\hline $\begin{array}{l}\text { 8. ¿Está de acuerdo con que si publica periódicamente su } \\
\text { contenido puede llegar a más clientes? }\end{array}$ & De acuerdo $65 \%$ \\
\hline $\begin{array}{l}\text { 9. ¿Está de acuerdo que es importante que su empresa tenga una } \\
\text { calendarización para llevar a cabo sus publicaciones? }\end{array}$ & De acuerdo $60 \%$ \\
\hline $\begin{array}{l}\text { 10. ¿Considera importante que se le dedique tiempo a la } \\
\text { creación y publicación de contenido? }\end{array}$ & De acuerdo $70 \%$ \\
\hline 11. ¿Está de acuerdo en que ofrecer ofertas aumentan las ventas? & De acuerdo $50 \%$ \\
\hline $\begin{array}{l}\text { 12. ¿Está de acuerdo en que reducir los precios pueden } \\
\text { aumentan las ventas? }\end{array}$ & Muy de acuerdo $50 \%$ \\
\hline 13. ¿Está de acuerdo que los servicios deben ofertarse? & En desacuerdo $40 \%$ \\
\hline 14. ¿Está de acuerdo en que las ofertas atraen a más clientes? & Muy de acuerdo 55\% \\
\hline $\begin{array}{l}\text { 15. ¿Está de acuerdo en que la decisión de compra de un cliente } \\
\text { se puede definir con una oferta? }\end{array}$ & Muy de acuerdo $40 \%$ \\
\hline $\begin{array}{l}\text { 16. ¿Considera que si publica sus servicios de fumigación en } \\
\text { redes sociales puede aumentar sus ventas? }\end{array}$ & Muy de acuerdo $50 \%$ \\
\hline $\begin{array}{l}\text { 17. ¿Considera que la publicación constante y oportuna de sus } \\
\text { servicios aumenten las ventas? }\end{array}$ & De acuerdo $50 \%$ \\
\hline $\begin{array}{l}\text { 18. ¿Considera que ofrecer ofertas de sus servicios de } \\
\text { fumigación ayude a aumentar las ventas? }\end{array}$ & De acuerdo $45 \%$ \\
\hline
\end{tabular}

Fuente: Elaboración propia. 
Los siguientes resultados se presentarán para hacer un analísis descriptivo de dicho estudio en el cual Se observa que un $70 \%(n=14)$ de las empresas de fumigación están "muy de acuerdo" en que publicar sus servicios de fumigación en redes sociales es importante, otro resultado que nos arrojo esta investigación fue que el 70\% ( $n=14)$ estuvieron "muy de acuerdo" en que publicar sus servicios en redes sociales atrae clientes, por otra parte el 55\% ( $\mathrm{n}=11)$ estuvieron "muy de acuerdo" en que las redes sociales aumentan sus ventas de servicios de fumigación, y también el 50\% $(n=10)$ consideran estar "de acuerdo" en que han aumentado sus ventas desde que ofrecen sus servicios en redes sociales.

De igual forma el 80\% ( $\mathrm{n}=16)$ esta "muy de acuerdo" en que las empresas de fumigación utilicen las redes sociales para aumentar sus ventas y no solo eso si no que el $75 \%(n=75)$ estuvo "de acuerdo" en que es importante publicar periódicamente contenido acerca de su empresa de fumigación, además de que el 50\% $(\mathrm{n}=10)$ estuvo "de acuerdo" en que periodizar el contenido de sus publicaciones ayuda a aumentar sus ventas.

Por otro lado el $65 \%(n=13)$ estuvo "de acuerdo" en que si publica periódicamente su contenido puede llegar a más clientes, tambien se observa que el 60\% $(n=12)$ está "de acuerdo" en que es importante que su empresa tenga una calendarización para llevar a cabo sus publicaciones, por otro lado el 70\% (n=14) estuvo "de acuerdo" en que es importante que se le dedique tiempo a la creación y publicación de contenido, en la variable ofertas se obsverva que un 50\% $(n=10)$ está "de acuerdo" en que ofrecer ofertas aumentan las ventas.

Otro de los resultados que se observaron es que el $50 \%(n=10)$ estuvo "muy de acuerdo" en que reducir los precios pueden aumentar las ventas, en la unica pregunta en la que el $40 \%(n=8)$ estuvo "en desacuerdo" fue en que los servicios deben ofertarse, por otro lado el 55\% ( $\mathrm{n}=11)$ estuvo "muy de acuerdo" en que las ofertas atraen a más clientes, de igual manera el 40\% (n=8) estuvo "muy de acuerdo" en que la decisión de compra de un cliente se puede definir con una oferta, ademas de que el 50\% (n=10) tambien estuvo "muy acuerdo" en que si publica sus servicios de fumigación en redes sociales puede aumentar sus ventas, tambien el 50\% (n=10) estuvo "de acuerdo" en que la publicación constante y oportuna de sus servicios aumentan las ventas, y por ultimo el $45 \%(n=45)$ estuvo de acuerdo en que ofrecer ofertas de sus servicios de fumigación ayude a aumentar las ventas. 
Con base a lo anterior, se hizo una transformación de las variables agrupando lo ítems en su respectiva variable a fin de poder explicar su relación, con relación a su media y desviación estándar Tabla 2. Parte de la relación que existe entre las variables en juego los datos descriptivos y con la relación a ello podemos mencionar que las redes sociales tenemos una media de 22.95 y una desviación estándar de 1.66938 siendo la variable que tiene mayor relevancia como se muestra.

Tabla 2. Estadísticos descriptivos

\begin{tabular}{|l|c|c|c|}
\hline & Media & Desv. Desviación & N \\
\hline Redes sociales & 229,500 & 166,938 & 20 \\
\hline Periodicidad & 202,500 & 204,875 & 20 \\
\hline Ofertas & 198,000 & 285,804 & 20 \\
\hline Aumento de ventas & 128,500 & 122,582 & 20 \\
\hline
\end{tabular}

Fuente: Elaboración propia.

La correlación bilateral de redes sociales sostien una signifiancia con ofertas y aumento de las ventas de .001, la periodicidad tiene relación con el aumento de las ventas de .003. las ofertas tienen una correlación con redes sociales de .001 y con aumento de venta de .003. por lo que en efecto el aumento de las ventas tiene correlacción importante con las variable independientes redes sociales, ofertas y periodicidad (tabla 3).

Una vez ya identificado que el análisis de componente muestra el modelo de regresión que se hizo mediante el análisis de la regresión se ha analizado la relación entre las variables criterio (aumento de ventas) y las variables independientes (redes sociales, periodicidad, ofertas).

Para ver si las empresas de fumigación generaban aumento de ventas a partir del uso de las redes sociales, la publicación periódica y la emisión de ofertas se condujo a hacer un análisis de regresión tabla 4 en donde los resultados para el resumen del modelo que permitió identificar las variables predictoras que explican el aumento de ventas de las empresas de fumigación. 
Tabla 3. Correlaciones

\begin{tabular}{|c|c|c|c|c|c|}
\hline & & $\begin{array}{c}\text { Redes } \\
\text { sociales }\end{array}$ & Periodicidad & Ofertas & $\begin{array}{l}\text { Aumento } \\
\text { de ventas }\end{array}$ \\
\hline \multirow{5}{*}{ Redes sociales } & Correlación de Pearson & 1 & $.481 *$ & $.671 * *$ & $.691 * *$ \\
\hline & Sig. (bilateral) & & 0,032 & 0,001 & 0,001 \\
\hline & $\begin{array}{l}\text { Suma de cuadrados y } \\
\text { productos vectoriales }\end{array}$ & 52,950 & 31,250 & 60,800 & 26,850 \\
\hline & Covarianza & 2,787 & 1,645 & 3,200 & 1,413 \\
\hline & $\mathrm{N}$ & 20 & 20 & 20 & 20 \\
\hline \multirow{5}{*}{ Periodicidad } & Correlación de Pearson & $.481 *$ & 1 & 0,288 & $.623 * *$ \\
\hline & Sig. (bilateral) & 0,032 & & 0,219 & 0,003 \\
\hline & $\begin{array}{l}\text { Suma de cuadrados y } \\
\text { productos vectoriales }\end{array}$ & 31,250 & 79,750 & 32,000 & 29,750 \\
\hline & Covarianza & 1,645 & 4,197 & 1,684 & 1,566 \\
\hline & $\mathrm{N}$ & 20 & 20 & 20 & 20 \\
\hline \multirow{5}{*}{ Ofertas } & Correlación de Pearson & $.671 * *$ & 0,288 & 1 & $.637 * *$ \\
\hline & Sig. (bilateral) & 0,001 & 0,219 & & 0,003 \\
\hline & $\begin{array}{l}\text { Suma de cuadrados y } \\
\text { productos vectoriales }\end{array}$ & 60,800 & 32,000 & 155,200 & 42,400 \\
\hline & Covarianza & 3,200 & 1,684 & 8,168 & 2,232 \\
\hline & $\mathrm{N}$ & 20 & 20 & 20 & 20 \\
\hline \multirow{5}{*}{$\begin{array}{c}\text { Aumento de } \\
\text { ventas }\end{array}$} & Correlación de Pearson & $.691 * *$ & $.623 * *$ & $.637 * *$ & 1 \\
\hline & Sig. (bilateral) & 0,001 & 0,003 & 0,003 & \\
\hline & $\begin{array}{l}\text { Suma de cuadrados y } \\
\text { productos vectoriales }\end{array}$ & 26,850 & 29,750 & 42,400 & 28,550 \\
\hline & Covarianza & 1,413 & 1,566 & 2,232 & 1,503 \\
\hline & $\mathrm{N}$ & 20 & 20 & 20 & 20 \\
\hline
\end{tabular}

*. La correlación es significativa en el nivel 0,05 (bilateral).

**. La correlación es significativa en el nivel 0,01 (bilateral).

El coeficiente de R de Pearson, nos identifica que existe una correlación significativa alta de $\mathbf{8 0 7}^{\mathbf{a}}$ entre las variables independientes redes sociales, periodicidad, ofertas con la dependiente aumento de ventas con un coeficiente de determinación de $\mathbf{R 2}=. \mathbf{6 5 1}$, indicando que el $65.1 \%$ de la variación observada es explicado por la combinación de redes sociales, periodicidad y ofertas. 
El error estándar es de .78903 por lo que los índices de aumento de ventas van a variar en una proporción de \pm .79 .

Por otro lado, el resultado de la prueba de Durbin Watson tiene una relación de 1.958, por lo que si el valor está entre 1 y 3 se acepta el supuesto e independencia.

Para el modelo de regresión que se prueba con los ítems de las variables predictoras como lo son redes sociales, periodicidad y ofertas nos explican el $65.1 \%$ de la varianza del ítem dependiente que es aumento de ventas $(\mathrm{R} 2=.651)$. Lo que indica que el aumento de las ventas se predice con las 3 variables.

En R2 ajustado el coeficiente sirve de control en cuestión de la sensibilidad cuando las muestras son de gran tamaño teniendo la R2 ajustado de $.586(58.6 \%)$ lo que pudiera considerarse un poco alejado del R cuadrado de .651 (65.1\%). A partir de los coeficientes se dice que el modelo está ajustado y cumple con los criterios estadísticos de aplicabilidad pues no se viola ningún supuesto.

Como se tiene literalmente que el estadístico $\mathrm{F}$ es el que permite contrastar la hipótesis nula de que el valor de la $\mathrm{R}$ es 0 , como $\mathrm{p}=.000<0.05$, teniendo en una sig. Cambio en $\mathrm{F}$ por lo que se puede aceptar que la hipótesis "la utilización de las redes sociales, las ofertas y la periodicidad aumenta las ventas del sector servicios en las empresas de fumigación" cumple con todas las características para decir que es aceptada.

Tabla 4. Resumen del modelo ${ }^{b}$

\begin{tabular}{|l|c|l|l|l|l|l|l|l|l|l|}
\hline \multirow{2}{*}{ Modelo } & $\mathbf{R}$ & \multirow{2}{*}{$\begin{array}{c}\mathbf{R} \\
\text { cuadrado }\end{array}$} & $\begin{array}{c}\mathbf{R} \\
\text { cuadrado } \\
\text { ajustado }\end{array}$ & $\begin{array}{c}\text { Esror } \\
\text { estándar } \\
\text { de la } \\
\text { estimación }\end{array}$ & $\begin{array}{c}\text { Cambio } \\
\text { en R } \\
\text { cuadrado }\end{array}$ & $\begin{array}{c}\text { Cambio } \\
\text { en F }\end{array}$ & gl1 & gl2 & $\begin{array}{c}\text { Sig. } \\
\text { Cambio } \\
\text { en F }\end{array}$ & $\begin{array}{c}\text { Durbin- } \\
\text { Watson }\end{array}$ \\
\hline 1 & $.807^{\mathrm{a}}$ & .651 & .586 & .78903 & .651 & 9.953 & 3 & 16 & .001 & 1.958 \\
\hline
\end{tabular}

a. Predictores: (Constante), Ofertas, Periodicidad, Redes_sociales

b. Variable dependiente: Aumento_de_ventas

Fuente: Elaboración propia basada en el programa SPSS versión 25.

Para el análisis del ANOVA (tabla 5) del modelo de regresión con las 4 variables se indica una predicción de la variable dependiente $\mathrm{VD}(\mathrm{F}=9.953$; p>.001) por lo tanto se puede contribuir a un modelo de regresión lineal.

Para construir el modelo se necesita una constante y un coeficiente que definan una ecuación de regresión, pues el coeficiente que corresponde a la constante en su origen de la recta de regresión (B0) y el coeficiente que corresponde a las variables independientes 
que son (redes sociales, periodicidad, ofertas) es la pendiente de la recta (B1) lo que indicara un cambio en la variable dependiente por cada cambio de la independiente que se mencione.

Por tal efecto se tiene por fórmula $\mathbf{y}=\mathbf{a}+\mathbf{b x}, \mathbf{y}$ sustituyendola quedaría de la siguiente manera.

$\mathrm{Y}=.588+.200$ redessociales $\mathrm{X}+.236$ periodicidadX +.146 ofertas $\mathrm{X}$

Tabla 5. Coeficientes ${ }^{a}$

\begin{tabular}{|c|c|c|c|c|c|c|}
\hline & \multirow{2}{*}{ Modelo } & \multicolumn{2}{|c|}{$\begin{array}{ll}\text { Coeficientes } & \text { no } \\
\text { estandarizados } & \end{array}$} & \multirow{2}{*}{$\begin{array}{l}\begin{array}{l}\text { Coeficientes } \\
\text { estandarizados }\end{array} \\
\text { Beta }\end{array}$} & \multirow[t]{2}{*}{$\mathbf{t}$} & \multirow[t]{2}{*}{ Sig. } \\
\hline & & B & Desv. Error & & & \\
\hline \multirow[t]{4}{*}{1} & (Constante) & .588 & 2.637 & & .223 & .826 \\
\hline & Redes_sociales & .200 & .160 & .272 & 1.250 & .229 \\
\hline & Periodicidad & .236 & .101 & .395 & 2.339 & .033 \\
\hline & Ofertas & .146 & .086 & .341 & 1.710 & 107 \\
\hline
\end{tabular}

a. Variable dependiente: Aumento_de_ventas

Fuente: Elaboración propia basada en el programa SPSS versión 25.

Por lo tanto se pueden realizar afirmaciones respecto a los resultados presentados en el coeficiente de Beta:

Por cada unidad que aumente las redes sociales, el aumento de las ventas crecerá en un .200 unidades.

Por cada unidad que aumente la periodicidad, el aumento de ventas crecerá en un .236 unidades.

Por cada unidad que aumente las ofertas, el aumento de ventas crecerá en un .146 unidades.

\section{CONCLUSIÓN O CONSIDERACIONES FINALES}

El establecimiento de las ventajas de las redes sociales como estrategia de comercialización en las empresas del sector servicios de fumigación se pudo comprobar que las redes sociales aumentan las ventas de las empresas de fumigación, de igual forma establecer una correlación significativa con cada una de las variables independientes como redes sociales, periodicidad y ofertas, comprobando que realmente son factores esenciales para lograr aumentar las ventas en las empresas de fumigación, de igual forma 
se pudieron determinar las futuras líneas de investigación que pudieran surgir a partir de esta investigación.

De acuerdo al objetivo que se planteó al inicio tal como "identificar si los factores como las redes sociales, la periodicidad y las ofertas aumentan las ventas del sector servicios de fumigación”, gracias a los resultados obtenidos y el análisis elaborado se pudo lograr los objetivos y reafirmarlos.

De igual forma la primer variable establecida las redes sociales se pudo afirmar que si aumentan las ventas dados los resultados obtenidos en el análisis, como lo refería Lucila (2018) de acuerdo a un estudio, el 72\% de los negocios con presencia en Facebook y Twitter afirma haber aumentado sus ventas gracias a la utilización y difusión de contenidos a través de ellas.

Con base a la segunda variable la cual es la periodicidad se pudo afirmar que la periodicidad con la que se realizan las publicaciones influye y causa un gran impacto tal y como lo dice la revista Merca 2.0 (s.f) "Es mejor realizar pocas publicaciones y cumplirlas, que ser muy activos, pero nada periódicos, ya que publicar a destiempo o con contenido de baja calidad perjudica la imagen de toda empresa o marca" con referencia a la revista Merca 2.0 y con los resultados obtenidos en esta investigación se puede decir que se cumple el supuesto de la variable de manera correcta.

Respecto a la tercera variable las ofertas, con referencia a Dolak (2012), define las promociones de venta como "Incentivos a corto plazo para incentivar las ventas de productos o servicios", con esto y con el análisis de los resultados favorables obtenidos se comprueba que las ofertas aumentan las ventas de las empresas de fumigación.

Por otro lado se puede encontrar que no solamente estos 3 factores pudieron ayudar al aumento de las ventas sino que también se detectó que por la pandemia la gente estuvo inmersa en la tecnología, esto mas que representar una amenaza para las empresas pudiera resultar una oportunidad ya que hubo un incremento de utilizacion de redes sociales de personas que no lo hacian de forma frecuente su uso, pudiendo captar mayor numero de clientes.

\section{LISTA DE REFERENCIAS}

Amiri, S., \& Woodside, J. M. (2017). Emerging markets: the impact of ICT on the economy and society. Digital Policy, Regulation and Governance, 19(5), 383- 
396. Recuperado de: https://www.emeraldinsight.com/doi/abs/10.1108/ DPRG04-2017-0013

Bricio Samaniego, K., Calle Mejía, J., \& Zambrano Paladines, M. (2018). El marketing digital como herramienta en el desempeño laboral en el entorno ecuatoriano: estudio de caso egresados de la Universidad de Guayaquil. Universidad y Sociedad, 10(4), 103-109. Recuperado de: http://rus.ucf.edu.cu/index.php/rus

Bernal, C. (2010). Metodología de la Investigación (Tercera Edición). Recuperado de: https://abacoenred.com/wp-content/uploads/2019/02/El-proyecto-deinvestigación-F.G.-Arias-2012-pdf.pdf

Cancela, G. Cea, R. Galindo, N. Valilla, G.(2010).Metodología de la Investigación Educativa. Universidad Autónoma de Madrid. Pág., 8. Recuperado de: http://www.uam.es/personal_pdi/jmurillo/InvestigaciónEE/Presentaciones/C urso_10/EX-POST-FACTO_Trabajo.pdf

Cognodata. (2019). ¿Qué es un análisis descriptivo? Recuperado de: https://www.cognodata.com/blog/que-es-analisis-descriptivo/

Gallego, J. (2010). Tecnologías de la información y de la comunicación. Recuperado de: https://recyt.fecyt.es/index.php/BORDON/article/view/29200

Giraldo, V. (2019). Descubre qué es la publicidad, para qué sirve y cómo influye en la sociedad. Recuperado de: https://rockcontent.com/es/blog/publicidad/

Genwords (2018). ¿Qué es el Social Media Marketing? Recuperado de: https://www.genwords.com/blog/social-media-marketing.

George, D. \& Mallery, P. (2003). SPSS for Windows step by step: A simple guide and reference. Recuperado de: https://wps.ablongman.com/wps/media/objects/385/394732/george4answers.pdf

Hernandez, R. Fernandez, C. Baptista, M. (2007). Metodologia de la investigación. Recuperado de: https://www.uca.ac.cr/wpcontent/uploads/2017/10/Investigacion.pdf

John, J.K. \& Kumar, N. (2015). Factors affecting consumers' perceptions of digital sales promotions - an experimental investigation. International Journal of science and Research. Vol. 4, Issue 4, p. 587-590.

Krum, C. (2010). Mobile Marketing, Finding Your Customer No Matter Where They Are. Recuperado de: 
https://www.researchgate.net/publication/318561405_Mobile_Marketing_A_Ne $\underline{\text { w_Direct }}$ Marketing_Promotional_Channel

Kirpatrick, D. (2011). El efecto Facebook. La verdadera historia de la empresa que esta conectando

al

Mundo.

http://scielo.sld.cu/scielo.php?script=sci_arttext\&pid=S2218$\underline{36202018000400103}$

Lamberton, C. Stephen, A.(2016). A Thematic Exploration of Digital, Social Media, and Mobile Marketing: Research Evolution from 2000 to 2015 and an Agenda for Future Inquiry. Journal of Marketing. Recuperado de:10.1509/jm.15.0415

Rodríguez, I. (2014). Marketing Digital y Comercio

Electrónico.http://scielo.sld.cu/scielo.php?script=sci_arttext\&pid=S2218$\underline{36202018000400103}$

Ramos, J (2016). Marketing de contenidos. Guia practica. 2da ed. S.L: Independly published.

Salas, A. (2020). ¿Qué es el marketing de contenidos?. Recuperado de: https://antoniosala.es/marketing-de-contenidos-ejemplos/

Sánchez, A. (2012) ANÁLISIS DE COMPONENTES PRINCIPALES.

Soengas, Vivar y Abuín (2015). Nuevas estrategias de publicidad y marketing para una sociedad hiperconectada. Recuperado de: https://telos.fundaciontelefonica.com/archivo/numero101/nuevas-estrategias-depublicidad-y-marketing-para-una-sociedad-hiperconectada/?output=pdf

Recuperado de: https://core.ac.uk/download/pdf/30046374.pdf

Thompson, I. (2015). Definición de Publicidad. Recuperado de:

Tamayo, M. (2005). El proceso de la investigación cientifica. Recuperado de:https://www.gob.mx/cms/uploads/attachment/file/227860/El_proceso_de_la investigaci_n_cient_fica_Mario_Tamayo.pdf

Vargas Sánchez, L. A. (2015). Marketing verde como alternativa económica - Luz Vargas. Revista GEON (Gestión, Organizaciones Y Negocios), 2(2), 140-143. Recuperado de: https://doi.org/10.22579/23463910.111 